\title{
Seizures and lost pulses: an atypical case of Takayasu's arteritis
}

\author{
Soumava Mukherjee $^{1}$, Avishek Naskar ${ }^{2}$, Atri Chatterjee ${ }^{3}$ \\ ${ }^{1}$ (General Medicine, Nil Ratan Sircar Medical College \& Hospital/West Bengal University of Health Sciences, \\ India) \\ ${ }^{2}$ (General Medicine, Nil Ratan Sircar Medical College \& Hospital/West Bengal University of Health Sciences, \\ India) \\ ${ }^{3}$ (Neuromedicine, Nil Ratan Sircar Medical College \& Hospital/West Bengal University of Health Sciences, \\ India)
}

\begin{abstract}
Seizure is a rare neurological manifestation of Takayasu's arteritis (TA). This report is about a 15 year old female patient, admitted with loss of consciousness, generalised seizures and hypertension. The patient had asymmetric peripheral pulses and unequal blood pressure in the arms. Doppler study showed diminished blood flow predominantly in left upper limb, while fluorescent aortogram revealed left subclavian artery stenosis. MRI was suggestive of posterior reversible encephalopathy syndrome (PRES). Electroencephalogram (EEG) report was consistent with cerebral dysrhythmia. The patient had coexistent ostium secundum atrial septal defect. Previous case reports attribute seizures to ischemia (caused by involvement of the carotid and vertebral arteries) and PRES in patients of TA. The presence of cerebral dysrhythmia after clinical improvement made the cause of seizure difficult to ascertain in our patient. The presence of atrial septal defect (ASD) was possibly coincidental.
\end{abstract}

Keywords: Atrial septal defect, cerebral dysrhythmia, Posterior reversible encephalopathy syndrome, Seizure, Takayasu's arteritis.

\section{Introduction}

Takayasu's arteritis (TA) is a granulomatous vasculitis of the large vessels, with a predilection towards branches of aorta. Patients commonly present with headache, visual symptoms and systemic features of fever, malaise, weight loss etc [1]. Commonly observed signs are hypertension, asymmetric pulses and bruits over large vessels [2]. Majority of Indian patients suffer from type III TA [3].

Constitutional symptoms are predominant in TA, but neurological features like headache (most common), stroke and hypertensive encephalopathy may also occur. Seizure is rarely reported in patients of TA [4]. This case is about a 15 year old female, presenting with generalised seizures, asymmetric peripheral pulses and systemic hypertension, whose MRI brain revealed features suggestive of PRES and EEG showed cerebral dysrhythmia, even after clinical improvement. So, this study implicates a rare association of seizure with TA. The study also reiterates the importance of systemic examination in a patient with seizure.

\section{Case presentation}

A 15 year old girl without any previous medical history presented to the emergency department with history of occipital headache since 2 months, vomiting with two episodes of seizures since 2 days followed by loss of consciousness. She suffered generalised seizures without fever or any previous episode of seizures. On examination in the emergency room, she had a regular pulse rate of 90 per minute, feeble radial, brachial and carotid pulses on left side with the rest of peripheral pulses being normal, blood pressure was $150 / 90 \mathrm{~mm}$ of $\mathrm{Hg}$ in right arm and 106/84 $\mathrm{mm}$ of $\mathrm{Hg}$ in left arm, respiratory rate of 16 per minute and $\mathrm{SpO}_{2}$ of $98 \%$ while breathing room air. Neurological examination revealed grimacing on painful stimulus, bilateral pale optic disc with blurred disc margin on fundoscopy, flaccid quadriparesis, bilateral Babinski sign without any sign of meningeal irritation. Cardiovascular examination showed loud first heart sound, wide fixed split second heart sound with signs of pulmonary hypertension. Patient was resuscitated and administered injection Sodium valproate $500 \mathrm{mg}$ twice daily and Labetalol $20 \mathrm{mg}$.

Laboratory reports revealed normal blood counts \& electrolytes, with elevated ESR $\left(120 \mathrm{~mm}\right.$ in $1^{\text {st }}$ hour). Patient regained consciousness after $24 \mathrm{hrs}$ of admission and had no further episode of seizures. A provisional diagnosis of large vessel vasculitis with seizure disorder attributable to hypertensive encephalopathy was reached and further investigations were planned.

MRI brain done on day 1 of hospital stay showed focal areas of hyperintensity in bilateral parietal, frontal and adjacent occipital region in T2 weighted FSE \& FLAIR images [Fig.1]. Diffusion weighted images showed mild restriction with hyperintensity on apparent diffusion coefficient (ADC) in the above areas. The imaging study was suggestive of white matter demyelination. 
An electroencephalogram done on day 4 of hospitalization revealed irregular bursts of sharp waves and slow wave discharges bilaterally in a background of slow alpha rhythm. Echocardiography showed ostium secundum type of atrial septal defect with mild pulmonary hypertension without reversal of the shunt. Doppler study revealed compromised blood flow predominantly in left upper limb due to continuous thickening of arterial wall. Fluorescent aortogram revealed left subclavian artery disease with $80 \%$ stenosis involving approximately $3 \mathrm{~cm}$ of its length, left renal artery was not visualised [Fig. 2]. Bilateral carotids, right subclavian artery, abdominal aorta were normal. Hence, a diagnosis of TA was made according to American College of Rheumatology criteria [5]. Mantoux test and VDRL were negative. Patient was put on oral prednisolone $1 \mathrm{mg} / \mathrm{kg} /$ day for 12 weeks along with Sodium valproate and Losartan. Patient was planned for left renal artery bypass grafting, but was lost to follow-up.

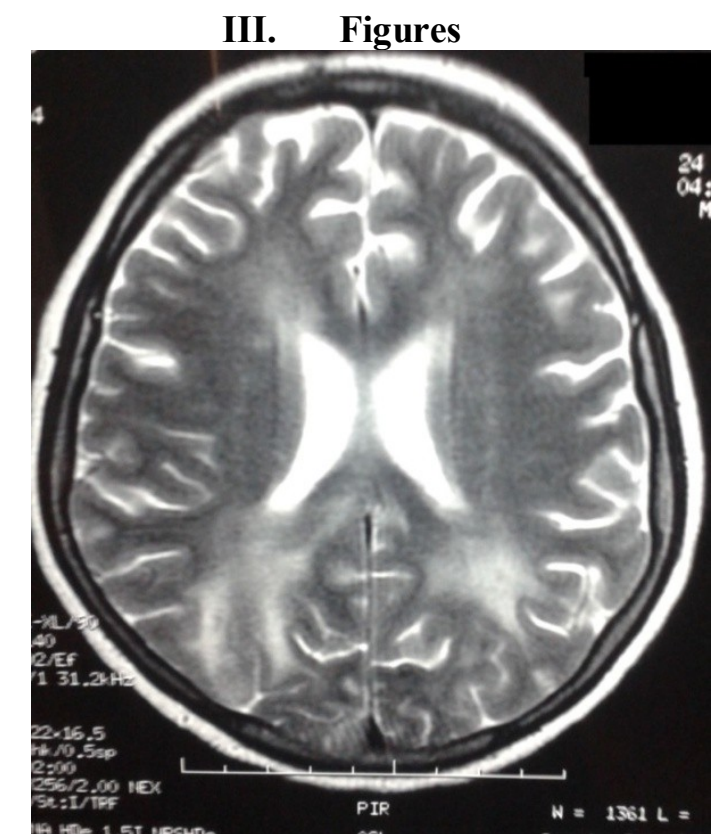

Figure 1: MRI brain showing white matter hyperintensity in bilateral parietal and adjacent occipital region in T2 weighted image

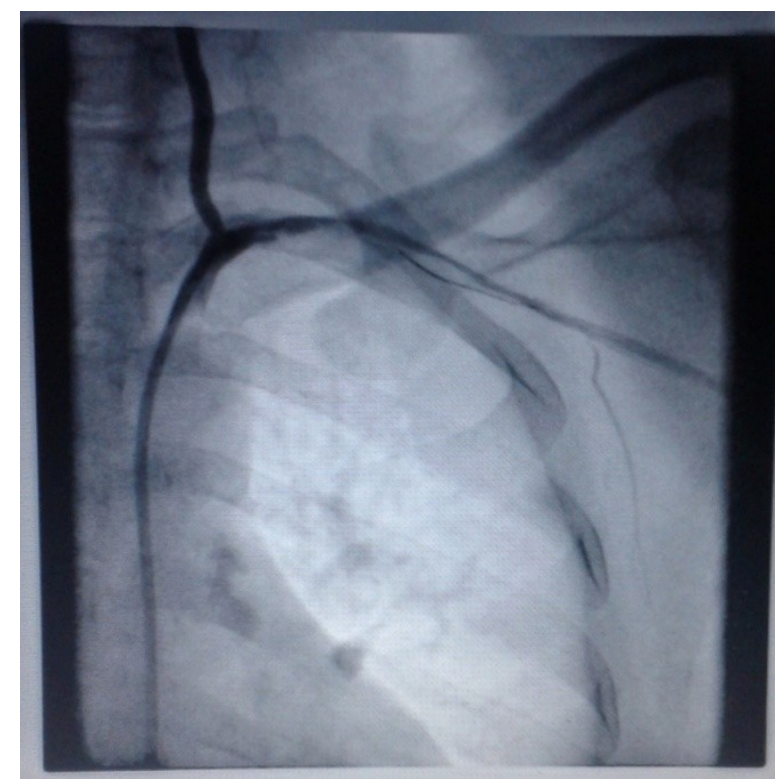

Figure 2: Aortogram showing left sublavian artery stenosis

\section{Conclusion}

Takayasu's arteritis (TA) or occlusive thromboaortopathy is an inflammatory arteritis of large vessels, predominantly affecting young females and involving the aorta and its branches. Though its aetiology is 
unknown, possible causes are autoimmune and infective, like tuberculosis and syphilis. Cases are most commonly reported from Japan, China, India and other south-east Asian countries.

Clinically TA may present with vascular claudication, hypertension, fever of unknown origin, stroke, bruit in large and medium sized arteries, unequal arm blood pressures and aortic regurgitation. Neurological manifestations have been found in $52.7 \%$ of TA [4]. Seizure has rarely been reported as a neurological complication of TA. Seizure associated with TA has been attributed to cerebral ischemia and hypertensive encephalopathy which is occasionally associated with PRES $[4,6]$. The symmetrical white matter hyperintensity in bilateral frontal, parietal and adjacent occipital regions present in the MRI are suggestive of PRES [7].

A case based review showed association of Takayasu's arteritis with PRES wherein all patients were females aged less than 40 years who had renovascular hypertension and presented with headaches and seizures [8]. However, in our patient cerebral dysrhythmia was present even after clinical features of PRES resolved. So it is difficult to ascertain the cause of seizure in our patient. Antiepileptic drugs have been reported to be effective in reducing the long term incidence of seizure in these patients [4]. The presence of ostium secundum ASD in our patient is possibly coincidental. However, seizures have been associated with ASD in patients with left ventricular hypertrabeculation [9], no evidence of which was found in echocardiography of the patient.

Treatment of TA includes immunomodulation, vascular intervention and control of hypertension. Immunomodulators like oral steroids and methotrexate (in patients with relapse) are therapy of choice. Vascular surgery is considered in patients with severe coronary or cerebral vessel disease, severe aortic regurgitation, critical limb ischemia, renal artery stenosis causing resistant hypertension and large aneurysms with risk of rupture [10]. In conclusion, our report not only presents a rare association, but also reiterates the importance of systemic examination of a patient presenting with seizures.

\section{References}

[1]. Johnston SL, Lock RJ, Gompels MM, Takayasu arteritis: Review, Journal of Clinical Pathology, 55(7), 2002, 481-486.

[2]. Sheikhzadeh A, Tettenborn I, Noohi F, Eftekharzadeh M, Schnabel A. Occlusive Thromboaortopathy (Takayasu Disease) : Clinical and Angiographic Features And a Brief Review of Literature, Angiology, 53(1), 2002, 29-40.

[3]. Sharma B.K, Sagar S, Singh AP, Suri S. Takayasu Arteritis in India, Heart \& Vessels, 7(1), 1992, $37-43$.

[4]. Ioannides MA, Eftychiou C, Georgiou GM, Nicolaides E. Takayasu Arteritis Presenting As Epileptic Seizures: A Case Report And Brief Review Of the Literature, Rheumatology International, 29(6), 2009, 703-705.

[5]. Arend WP, Michel BA, Bloch DA, Hunder GG, Calabrese LH, Edworthy SM, et. al. The American College of Rheumatology 1990 criteria for the classification of Takayasu arteritis, Arthritis \& Rheumatology, 33(8), 1990, 1129-1134

[6]. Zaki SA, Chavan V, Shanbag P. Unusual presentation of Takayasu's arteritis as posterior reversible encephalopathy syndrome, Annals of Indian Academy of Neurology, 14(3), 2011, 214-216

[7]. Magana SM, Matiello M, Pittock SJ, McKeon A, Lennon VA, Rabinstein AA et. al. Posterior reversible encephalopathy syndrome in neuromyelitis optica spectrum disorders, Neurology, 72(8), 2009, 712-717

[8]. Camara-Lemarroy CR, Lara-Campos JG, Perez-Contreras E, Rodriguez-Gutierrez R, Galarza-Delgado DA. Takayasu's arteritis and posterior reversible encephalopathy syndrome: a case-based review, Clin Rheumatol, 32(3), 2013, 409-415

[9]. Nagel B, Gruber-Sedlmayr U, Uhrig S, Stollberger C, Klopocki E, Finsterer J. Left ventricular hypertrabeculation/noncompaction with epilepsy, other heart defects, minor facial anomalies and new copy number variants, BMC Med Genet, 13, 2012, 60

[10]. Perera AH, Mason JC, Wolfe JH. Takayasu arteritis: Criteria for surgical intervention should not be ignored, Int $\mathrm{J}$ Vasc Med 2013;Article ID 618910 